\title{
Neuroprotection In Acute Stroke
}

Ali Amini Harandi ,.

Department of Neurology, Shahid Beheshti University of Medical Sciences, Tehran, Iran.

*Corresponding Author: Email: amini_alli@yahoo.com

Stroke is the leading cause of adult disability and remains the third most common cause of death in industrialized nations. The concept of neuroprotection mainly came from the studies of the pathology and pathophysiology of ischemic brain injury. Greater understanding of the pathophysiology of neuronal damage in ischemic stroke has generated interest in neuroprotection as a management strategy. Neuroprotective agents in stroke treatment, have generated long-term interest that have the potential to preserve brain tissue and improve overall outcome. One arm of neuroprotective agents limits acute injury to neurons in the ischemic penumbra. Many of these agents modulate neuronal receptors to reduce release of excitatory neurotransmitters, which contribute to early neuronal injury. Other neuroprotective agents prevent potentially detrimental events associated with return of blood flow. Returning blood contains leukocytes that may occlude small vessels and release toxic products. In fact they should act targeting excitotoxicity, oxidative and nitrosative stress, and inflammation. The past few decades have produced a plethora of negative neuroprotective trial results. The questions of feasibility and practicability cannot be resolved simultaneously. In the future, optimal therapy may be achieved by combining neuroprotective agents with complementary mechanisms. Relevant areas of interest include the search for safe and effective treatment strategies that combine neuroprotection reperfusion, better use of advanced brain imaging for patient selection, and wider implementation of prehospital conducted clinical trials.

Key words: Stroke, disability, Neuroprotection

DOI: $10.7575 /$ aiac.abcmed.ca1.47

Published Date: February 2017

Peer-review is under responsibility of the 9th Iranian Stroke Congress.

Published by Australian International Academic Centre, Australia

This published work is open access under the CC BY license.

Available online at www.abcmed.aiac.org.au 